\title{
Application of a Porous Media Model for the Acoustic Damping of Perforated Plate Absorbers
}

Jianguo Wang*, Philip Rubini*, Qin Qin

School of Engineering and Computer Science, University of Hull, Kingston upon Hull, HU6 7RX, UK

Nomenclature

Latin Letters

\author{
Area of an Orifice \\ Speed of Sound \\ Bias Flow Resistance Coefficient \\ Discharge Coefficient \\ Diameter of Perforation \\ Frequency \\ Enthalpy \\ Wave Number \\ Cavity Depth \\ Acoustic End Correction Length
}

Acoustic Effective Thickness of a Plate

Real Thickness of a Plate

Flow Mach Number in Perorations

Bias Flow Mach Number

Pressure

Acoustic Signal Amplitude

Resonance Parameter

Normalzied Specific Acoustic Resistance

Radial direction of a Cylindrical Coordinate System

Radius of Perforations

Bias Flow Induced Normalzied Specific Resistance

Reflection Coefficient

Momentum Source Term

Momentum Source Term in Porous Media Model

Time

Temperature

Velocity Component in $x$ direction

Physical Velocity in the Porous Media Region

Velocity in Axial and Radial Directions

Acoustic Particle Velocity in Orifices

Mean Bias Flow Velocity in Orifices

Particle Velocity

Normalized Specific Acoustic Reactance

Coordinate Location in $x$ Direction

Normalized Specific Acoustic Impedance

Specific Acoustic Impedance

Greek Symbols
Absorption Coefficient
Porosity of Perforated Plate
Dynamic Viscosity of Air 


\author{
Kinematic Viscosity of Air ${ }^{1}$ \\ Denstiy of Air \\ Viscous Stress \\ Fok's Function \\ Angular Frequency \\ Diffusion Coefficient
}

\section{Abstract}

Perforated panel, or plate, absorbers are commonly employed to reduce sound pressure levels across a broad range of applications including the built environment, industrial installations and propulsion devices. The acoustic performance of a perforated plate absorber depends upon a number of parameters such as physical geometry of the absorber, acoustic spectrum and sound pressure level of the acoustic source. As a consequence, experimental determination of acoustic properties is often required on an individual basis in order to optimise performance.

Computational simulation of a perforated plate absorber would alleviate the necessity for experimental characterisation. Fundamentally this can be achieved by the direct numerical solution of the underlying governing equations, the compressible form of the Navier-Stokes equations. The numerical methodology is available and routinely implemented as a Computational Fluid Dynamics solver. However, the numerical simulation of flow through a perforated plate with a large number of very small orifices would require significant computational resource, not routinely available for engineering design simulations.

In this paper, a porous media model, implemented as a sub-model within a CFD solver, is investigated and validated against a number of well-acknowledged acoustic experiments undertaken in an impedance tube, for a sound pressure wave incident normal to a perforated plate. The model expresses the underlying governing equations within the perforated plates in terms of a pseudophysical velocity representation. Comparison between three dimensional, compressible, laminar flow CFD simulations and experimental data, demonstrate that the porous model is able to represent acoustic properties of perforated plate absorbers in linear and non-linear absorption regimes and also the inertial effect in the presence of a mean bias flow.

The model significantly reduces the computational resource required in comparison to full geometric resolution and is thus a promising tool for the engineering design of perforated plate absorbers.

Keywords: Perforated Plate Absorber; Porous Media Model; Numerical Simulation;

\section{Introduction}

This work was originally motivated by a requirement to control pressure oscillations in a gas turbine combustion chamber, when operating under lean, premixed conditions. Fuel and air are premixed upstream of the combustor to avoid the formation of stoichiometric regions in lean premixed combustion. Therefore, lean premixed combustion reduces maximum reaction zone temperatures and consequently reduces nitrogen oxide (NOx) pollutant emissions. Unfortunately, lean premixed combustion is much more susceptible to combustion instability $(1,2)$, the resultant pressure oscillations

\footnotetext{
$1 *$ Corresponding author.

Email addresses: ethan19900428@gmail.com, P.A.Rubini@hull.ac.uk
} 
can be of such magnitude to result in significant structural damage.

The combustion chamber liner of a gas turbine engine requires cooling, which historically has been achieved by directing compressor delivery air around the exterior of the liner, and through cooling slots(3). Recent developments in cooling technology have introduced the use of perforated wall and effusion cooling (4). This in turn has motivated a renewed interest in the ability of a perforated plate to attenuate pressure fluctuations $(5,6)$.

A considerable literature exists, reporting experimental studies in which the acoustic properties of perforated liner absorbers are influenced by a variety of flow and geometry factors. Among these influencing factors, important geometric factors include plate porosity (7-9), liner thickness $(5,10)$, perforation geometry (11-13), and geometry of the cavity (14-16). Important flow factors include incident sound pressure level (17-19), bias flow and grazing flow speeds (10, 20-23). Despite the considerable range of investigations, the majority have focused on only one or two influencing factors and in many instances the configuration of the test rig and scope of measured parameters are often inconsistent between individual measurement datasets. In addition, the measured acoustic properties of a perforated liner are dependent upon the periphery acoustic boundary conditions, dimension and position of the perforated plates within the test rig. As a consequence, acoustic properties of a perforated liner absorber can significantly vary under different conditions and must be tested on a case by case basis.

The majority of previous CFD investigations into perforated plate absorbers have concentrated upon determining noise attenuation properties by resolving the detailed geometry of the perforations themselves (24-28). Such an approach is useful in providing data for model validation and understanding of noise dissipation mechanisms such as vortex shedding. However, there are often hundreds or thousands of small holes in a gas turbine film cooling perforated liner and the computational cost of resolving every individual hole would be computationally very expensive. One approach would be to resolve a reduce area of a perforated plate and to apply appropriate periodical boundary conditions $(24,29)$. This approach is unfortunately not applicable for the simulation of cylindrical combustor liners under complex flow conditions such as those in gas turbine combustors. Therefore full geometric resolution remains computationally expensive for the simulation of gas turbine combustor liners at realistic flow conditions for routine engineering design.

Lahiri (30) documented a detailed investigation into the effect of a wide range of factors which influence the acoustic performance of perforated plate absorbers. He found that details of the perforation geometry such as the shape of perforation cross section and the perforation pattern had an almost negligible influence on the overall attenuation effect. Similar conclusions were obtained by a number of other investigations including $(11,12,31,32)$. Therefore, empirical evidence supports the hypothesis that it would be feasible to model a perforated plate as a homogenous region without necessitating full geometric resolution.

In this paper a perforated plate is represented by an empirical, Physical Velocity, Porous Media Model (PVPM Model). It will be demonstrated that this PVPM model is able to substantially reduce the computational resource required in comparison to that required by traditional methods of resolving detailed hole geometries. The capability of the porous media model to represent the acoustic properties of perforated plate absorbers is validated in the linear absorption regime, non-linear absorption regime and in the presence of a mean bias flow. The aim of the paper is thus to propose and validate a method of using porous media that could be used as a computationally efficient tool for the engineering design of perforated plate absorbers. 
2 Methods

\subsection{Navier-Stokes Equations}

Computational Fluid Dynamics (CFD) is based upon the numerical solution of the underlying governing equations for fluid flow, under specified boundary conditions. The propagation of an acoustic plane wave in an impedance tube, incident normal to a perforated plate may be represented as an unsteady compressible laminar flow. The governing equations may be expressed, for brevity, in axisymmetric cylindrical polar coordinates as :

$$
\begin{gathered}
\frac{\partial \rho}{\partial t}+\frac{\partial\left(\rho u_{x}\right)}{\partial x}+\frac{1}{r} \frac{\partial\left(\rho r u_{r}\right)}{\partial r}=0 \\
\frac{\partial\left(\rho u_{x}\right)}{\partial t}+\frac{1}{r} \frac{\partial\left(\rho r u_{x} u_{x}\right)}{\partial x}+\frac{1}{r} \frac{\partial\left(\rho r u_{r} u_{x}\right)}{\partial r}=\frac{\partial \tau_{r x}}{\partial r}-\frac{\partial p}{\partial x}+S_{x} \\
\frac{\partial\left(\rho u_{r}\right)}{\partial t}+\frac{1}{r} \frac{\partial\left(\rho r u_{x} u_{r}\right)}{\partial x}+\frac{1}{r} \frac{\partial\left(\rho r u_{r} u_{r}\right)}{\partial r}=\frac{\partial \tau_{x r}}{\partial x}-\frac{\partial p}{\partial r}+S_{r} \\
\frac{\partial(\rho h)}{\partial t}+\frac{1}{r} \frac{\partial\left(\rho r u_{x} h\right)}{\partial x}+\frac{1}{r} \frac{\partial\left(\rho r u_{r} h\right)}{\partial r}=\frac{1}{r} \frac{\partial}{\partial x}(r \Gamma \partial \mathrm{h} / \partial \mathrm{x})+\frac{1}{r} \frac{\partial}{\partial r}\left(\mathrm{r} \Gamma \frac{\partial h}{\partial r}\right)+S_{h}
\end{gathered}
$$

where $x, r$ are the axial and radial directions of the cylindrical coordinate system and $u_{x}, u_{r}$ are the axial and radial velocities. $\tau_{r x}$ is the axial direction viscous stress due to flow velocity gradient in radial directions. $S_{x}, S_{r}$ are momentum source terms and $\mathrm{S}_{\mathrm{x}}=-\left(\mathrm{C}_{\mathrm{v}} \mathrm{u}_{\mathrm{x}}+\mathrm{C}_{\mathrm{i}} \mathrm{u}_{\mathrm{x}}^{2}\right)$ where $\mathrm{C}_{\mathrm{v}}$ and $\mathrm{C}_{\mathrm{i}}$ are viscous resistance and inertial resistance respectively. The ideal gas equation of state, $P=\rho R T$, is assumed to hold for determination of the fluid density.

\subsection{Physical Velocity Porous Media Model (PVPM Model)}

The specific acoustic impedance, $\mathrm{Z}$, of a perforated plate is defined by the driving pressure drop and the resultant particle velocity across the plate. The pressure drop across an individual orifice may be attributed to flow resistance effects acting on the through flow. Therefore the acoustic impedance of a perforated plate may be determined by appropriate representation of the inertial and viscous resistance terms as a function of the flow velocity. Historically the geometry and flow details within a porous zone have largely been beyond the capability of computer resources at the time, necessitating an approach whereby the porous zone is modelled as a homogenous region. This has been a common practice in CFD simulation of porous media flows. A PVPM model has been demonstrated to be able to successfully represent the mean flow velocity in a porous zone while ignoring perforation details (33-36). The PVPM model was originally developed for the representation of uniform and highly porous material which has a rigid frame and it will be used in this paper to capture acoustic damping effect of perforated plate absorbers.

The PVPM model introduces the porosity of a material, $\sigma$, into the governing equations. For example, when considering only the axial direction within a porous zone, the continuity and axial momentum equations may be written as:

$$
\frac{\partial(\sigma \rho)}{\partial t}+\frac{\partial\left(\rho \sigma u_{p x}\right)}{\partial x}+\frac{1}{r} \frac{\partial\left(\rho r \sigma u_{p r}\right)}{\partial r}=0
$$




$$
\begin{gathered}
\frac{\partial\left(\rho \sigma u_{p x}\right)}{\partial t}+\frac{\partial\left(\rho \sigma u_{p x} u_{p x}\right)}{\partial x}+\frac{\partial\left(\rho \sigma u_{p r} u_{p x}\right)}{\partial r}=\frac{\partial\left(\sigma \tau_{r x}\right)}{\partial r}-\frac{\partial(\sigma p)}{\partial x}+\sigma S_{p x} \\
\frac{\partial(\rho h)}{\partial t}+\frac{1}{r} \frac{\partial\left(\rho \sigma r u_{x} h\right)}{\partial x}+\frac{1}{r} \frac{\partial\left(\rho \sigma r u_{r} h\right)}{\partial r}=\frac{1}{r} \frac{\partial}{\partial x}(\sigma r \Gamma \partial h / \partial x)+\frac{1}{r} \frac{\partial}{\partial r}\left(\sigma r \Gamma \frac{\partial h}{\partial r}\right)+\sigma S_{p h}
\end{gathered}
$$

Comparison between the continuity equation (1) for non-porous region and equation (5) for the porous region demonstrates how the velocity magnitude must increase by a factor $1 / \sigma$ for mass to be conserved at the entrance of the porous zone, $u_{p x}=u_{x} / \sigma$. The magnitude of this physical velocity, $u_{p x}$, is identical to the average flow velocity in a real perforation which makes the acquisition of acoustic impedance possible. The velocity magnitude is however assumed to be homogenous everywhere in the porous media region. It is therefore not an exact representation of flow conditions within the real perforations. The magnitude of this assumption will be assessed as part of the validation of the model in which a number of well-acknowledged experiments are reproduced in order to determine if the model is able to represent acoustic properties of perforated plate absorbers in linear and non-linear absorption regimes and also the inertial effect in the presence of a mean bias flow.

\subsection{Inclusion of Inhomogeneity Effects}

A perforated plate may be represented by the porous media model as a layer of a homogenous material. However a number of important acoustic behaviours are not immediately represented by a homogenous model.

The pressure loss due to viscous effects within perforations is directly related to the geometry of perforations and this is lost by the homogenous geometry assumption. The viscous term is negligible in the presence of mean bias flows when the Reynolds number of the orifice flow becomes significant and viscous effects are overwhelmed by inertial effects. However, the viscous effect is important for the flow through a hole at low Reynolds number in the absence of mean bias flow. In this work, the viscous effect is ignored in cases where mean bias flow is present as this term becomes negligible, otherwise in cases with zero mean bias flow, the steady-state viscous resistance is approximated by the Hagen-Poiseuille equation (37) and is included as a viscous resistance term in the momentum source term in equation (6). The unidirectional perforations indicate anisotropic porosities within perforated plates. This is ensured by imposing a flow resistance in lateral directions of several order magnitudes greater than that in the axial direction so the flow only develops in the axial direction.

Empirical evidence demonstrates that the acoustic effective length of a short cylinder is greater than the true physical length due to acoustic radiation. This effect cannot be directly represented by the homogenous porous model and must therefore to be empirically modelled. The effective thickness of an orifice through an infinite large plate without the presence of mean flow was analytically derived by Ingard (38) and Rayleigh (39):

$$
l_{\text {eff }}=l_{\mathrm{t}}+8 r / 3 \pi \approx l_{\mathrm{t}}+0.85 d
$$

In later work it was shown that the end correction length may be reduced by acoustic interaction between neighboring holes, referred to as a hole-hole interaction effect. $(38,40)$. Melling $(41)$ analyzed the hole-hole interaction effect by referring to Fok's function $\Psi(40)$.

$$
\Psi=1-1.4092 \sqrt{\sigma}+0.33818 \sigma^{1.5}+0.06793 \sigma^{2.5}
$$

High particle velocity or mean bias flow in the orifice was additionally found to reduce the end correction effect $(42,43)$. Maa $(43)$ proposed a simple extra correction term due to high orifice velocities: 


$$
l_{\mathrm{eff}}=l_{\mathrm{t}}+0.85 d \Psi\left(1+\frac{\mathrm{M}}{\sigma}\right)^{-1}
$$

where $\mathrm{M}$ is the neck particle Mach number. It is common practice to assume that the high particle velocity and the mean bias flow reduce end correction due to the same physical mechanism $(30,44$, 45), therefore the neck particle Mach number in equation (10) can be replaced by the neck flow Mach number:

$$
\mathrm{M}=\frac{\left[U_{\mathrm{b}}+\sqrt{2} \mathrm{RMS}\left(U_{\mathrm{ac}}\right)\right]}{\mathrm{c}}
$$

In order to represent the acoustic effective thickness of a perforated plate, equation (10) and equation (11) are applied to replace the physical thickness when defining the geometry. This typically results in an end correction of order $1 \mathrm{~mm}$ for a plate with $3 \mathrm{~mm}$ diameter holes.

\subsection{Boundary Conditions, Numerical Scheme and Computational Grid}

Well imposed acoustic boundary conditions are critical if a CFD simulation is to be able to accurately represent an acoustic field in the computational domain. Such acoustic boundary conditions can be achieved either by expanding the calculation domain until acoustic reflections from the calculation domain boundaries exert little impact on near-field acoustics, or by defining boundary acoustic impedance properties. Acoustic impedance of boundaries is often not a priori parameter. In this paper, the first approach of expanding boundaries is adopted if acoustic impedance of boundaries is unknown. On the other hand, a general non-reflecting boundary condition proposed by Poinsot et. al. (46) and Selle et.al. (47) is applied to represent anechoic boundaries.

Best practice suggested that two or three layers of structured hexahedral meshes should be employed to represent the thickness of the perforated plate in preference to unstructured tetrahedral meshes. A number of additional criteria were required to be satisfied. Firstly, in order to accurately represent the geometry of the test rig at least 20 layers of grid cells were defined to resolve the radial direction of a flow duct. Secondly, a minimum of 25 time steps were required to resolve one time period of the physical input signal of the highest frequency and a minimum of 25 layers of grid cells were defined to resolve one wavelength of the incident signal of the highest frequency. Thirdly, the computational grid and solution time step were adjusted to ensure that the local flow Courant number was close to unity throughout the calculation domain.

The simulations were undertaken using the commercial CFD code, Ansys Fluent (Ansys Fluent, 2009), where a PVPM model is available. This model has not been previously validated for acoustic simulation through a perforated plate. All simulations were represented as three dimensional, compressible, laminar, time dependent, flow phenomena. Wherever possible, second order accurate approximations were selected. The pressure gradient term was resolved using a second order accurate scheme; all other spatial terms were approximated by a second order upwind numerical scheme. A bounded second order implicit temporal scheme was adopted to capture the small but rapidly changing acoustic pressures. The SIMPLE pressure-velocity correction algorithm was employed.

A porous material is typically consists of intertwining solid frames and void spaces. Void fluid spaces would normally require detailed resolution in a CFD simulation. However, meshing and resolving of all those very small void spaces are nearly impossible due to the huge amount of geometry details and the consequential computational cost. From another perspective, researchers and industry are often more interested in the macroscopic effect of porous region on the transverse flow rather than flow field details in each individual void space (48-50).

This work proposes that a perforated liner is able to be treated as a homogenous porous media region. 
Geometry details are ignored and the flow is assumed to be uniform in the porous media region. This method is able to significantly reduce the computational cost otherwise needed in resolving the large number of effusion cooling holes on a combustor liner. The number of meshes required resolving every individual hole and that required by treating the perforated plate as a homogenous porous zone is compared in Figure 1 . In this example, the plate is a $3 \mathrm{~mm}$ thick $0.1 \mathrm{~m} \times 0.1 \mathrm{~m}$ square plate with a number of perpendicular holes $(d=2.5 \mathrm{~mm})$ as shown in Figure $2(\mathrm{~b})$. When the plate is treated as a homogenous zone, with the cross section represented by a $20 \times 20$ grid and two grid layers across the thickness, this results in a total number of 800 cells in the porous media zone. In case where the real geometry of each hole is resolved, then each hole is represented by typically 12 cells across the radial direction and 10 cells in the axial direction. Such resolution is necessary as a minimum to represent the internal flow field. The mesh counts grow linearly with the number of perforations and as a result, the amount of computational time saved by using the porous media model becomes significant where very large numbers of small holes are to be resolved. The above argument does not include those fine meshes which are necessary around the exterior of the holes for grid consistency, which, if taken into account, will increase the mesh count ratio even further.

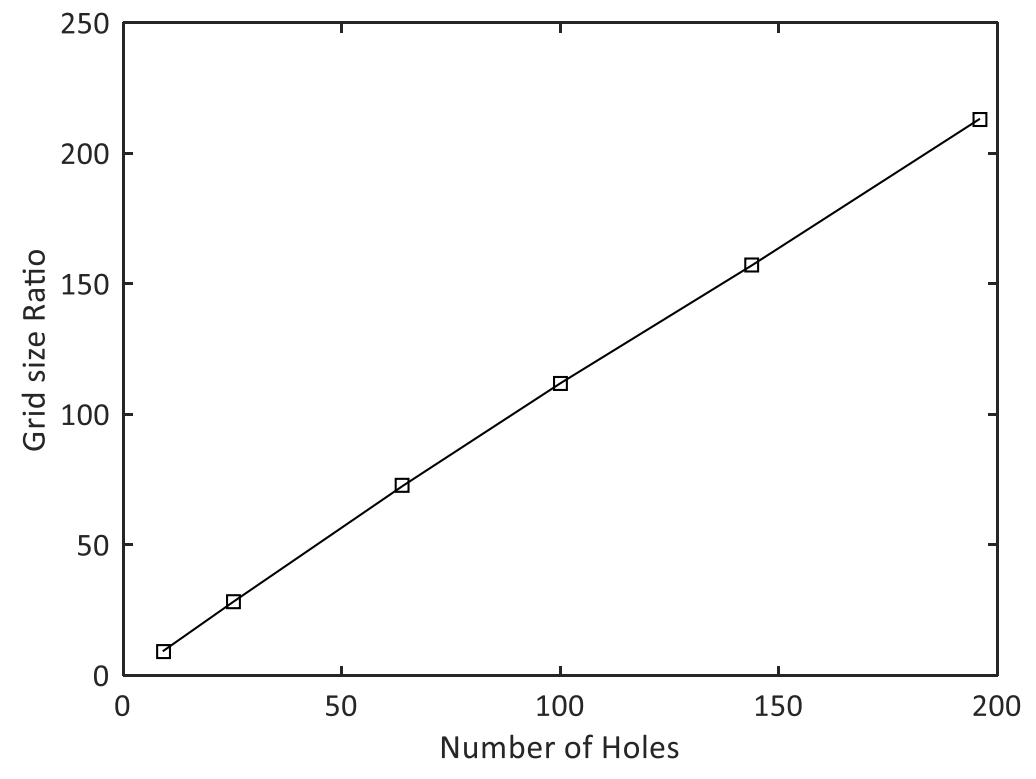

Figure 1 Ratio of grid resolution required for geometric resolution of perforations to that required using the PVPM model

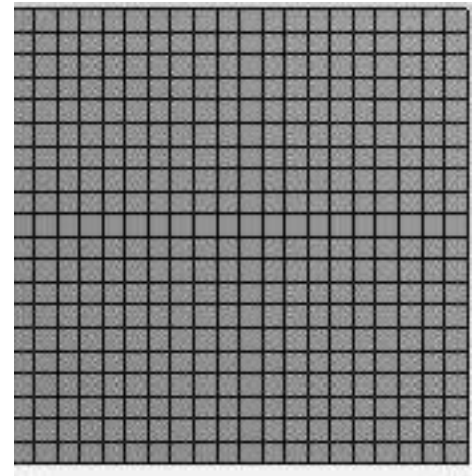

(a)

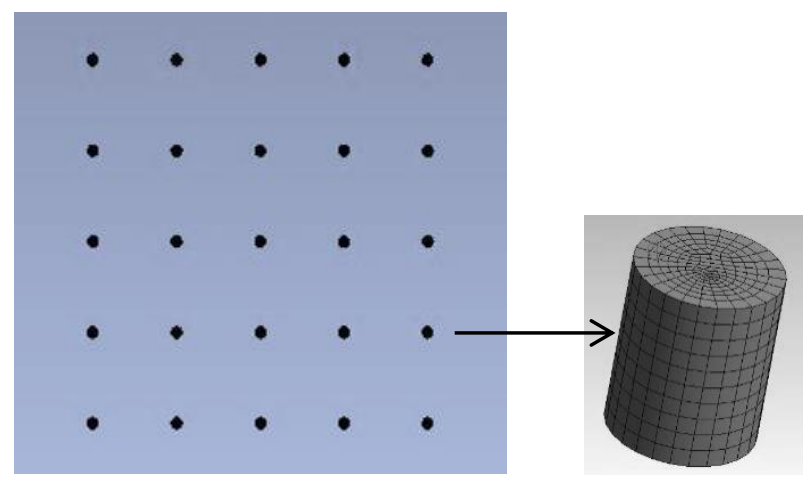

(b)

(c)

Figure 2 Two different meshing and calculation strategies for perforated plate region: (a) treating perforated plate as a porous media region; (b) calculation of every individual perforation; (c) a closeup of the meshes in a perforation 


\subsection{Two Microphone Method in Impedance Tubes}

The data collecting and processing strategy for the CFD simulations followed those applied in the validation experiments. The two-microphone method (51) was applied in many experiments cited in this work $(7,10,41)$. Figure 3 shows a typical impedance tube setup using two-microphone method. Two microphones are installed in front of the test sample with distances of $x_{1}$ and $x_{2}$ respectively.

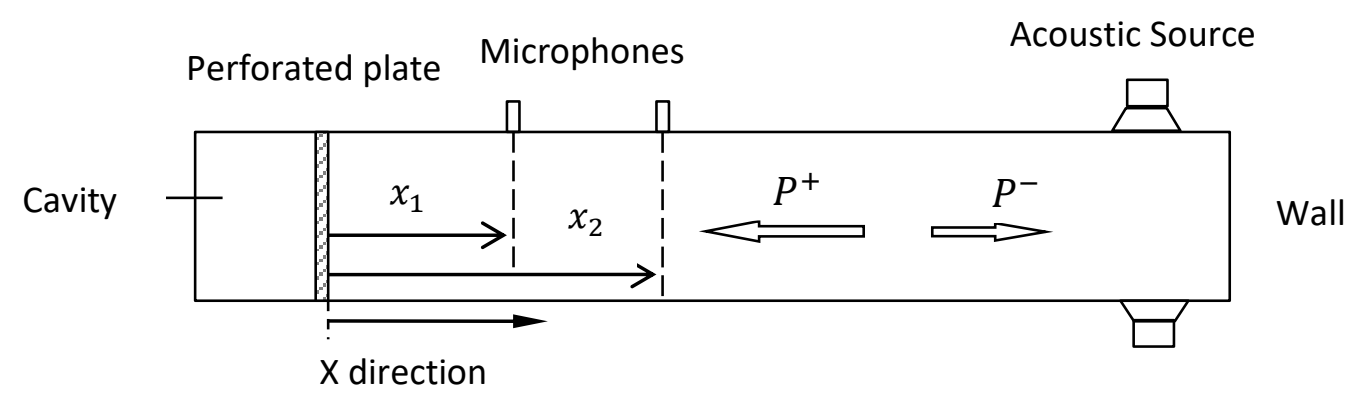

Figure 3 An acoustic experiment setup in an impedance tube using two-microphone method

Pressures at the microphones are the superimposition of two acoustic pressures travelling in the positive $X$ direction and negative $X$ direction and pressure at the two microphones at a given instant is:

$$
\begin{aligned}
& p_{1}=P^{+} \exp \left(i k x_{1}\right)+P^{-} \exp \left(-i k x_{1}\right) \\
& p_{2}=P^{+} \exp \left(i k x_{2}\right)+P^{-} \exp \left(-i k x_{2}\right)
\end{aligned}
$$

Solve for amplitudes of the waves $P^{+}$and $P^{-}$, results in:

$$
\begin{gathered}
P^{+}=\frac{p_{1} \exp \left[i k x_{1}\right]-p_{2} \exp \left[i k x_{2}\right]}{\exp \left(2 i k x_{1}\right)-\exp \left(2 i k x_{2}\right)} \\
P^{-}=\frac{p_{1} \exp \left[-i k x_{1}\right]-p_{2} \exp \left[-i k x_{2}\right]}{\exp \left(-2 i k x_{1}\right)-\exp \left(-2 i k x_{2}\right)}
\end{gathered}
$$

From which the acoustic reflection coefficient of the panel absorber may be obtained:

$$
R=\frac{P^{-}}{P^{+}}
$$

At the test sample surface $(x=0)$, acoustic pressure and acoustic velocity as a result of superimposition of the incident wave and the reflected wave are respectively :

$$
p=P^{+}+P^{-} ; v=\frac{P^{+}}{\rho c}-\frac{P^{-}}{\rho c}
$$

Therefore, the normalized specific acoustic impedance of the panel absorber is:

$$
z=r+i x=\frac{p}{v \rho c}=\frac{P^{+}+P^{-}}{P^{+}-P^{-}}=\frac{1+R}{1-R}
$$

where $r$ is the normalized specific acoustic resistance which is a measure of opposition that the perforated plate absorber presents to the acoustic flow resulting of the acoustic pressure, $x$ is the 
normalized specific acoustic reactance which describes the phase difference between the driving pressure difference and the resultant orifice velocity. The acoustic energy that is not reflected by the absorber is absorbed by the perforated plate absorber and the energy absorption coefficient is:

$$
\alpha=1-|R|^{2}=\frac{4 r}{(1+r)^{2}+x^{2}}
$$

As can be seen from equation (19), the optimum absorption effect of a perforate plate absorber takes place at the frequency where acoustic reactance is zero.

The two-microphone method calls for prior microphone calibration to match the phase and magnitude response of the two microphones. High precise calibration of microphones is however sometimes very difficult to achieve. Another simplified one-microphone transfer function method has been proposed by Chu (52) where only a single microphone is required to sequentially collect pressures at two different locations. Microphone calibration is avoided by using this method and this method is applied in the experiment by Jing and Sun (17) which is chosen as the validation experiment.

\section{Experiments Used For Validation}

\subsection{Linear Absorption Regime}

Validation of the PVPM model commenced with the study of the acoustic attenuation properties of perforated plate absorbers in the linear absorption regime. In this regime the sound pressure level (SPL) is such that in the acoustic resistance of a plate barely changes with incident SPL. The experiment of Lee and Kwon (7) was re-visited by the proposed CFD method in order to investigate effects of porosity, multi-layer perforated panels and the effect of an array of perforated plates. The experiment employs an impedance tube, similar to that shown in Figure 3 in which four perforated plates were tested in a $1 \mathrm{~m}$ long $60 \mathrm{~mm} \times 60 \mathrm{~mm}$ square impedance tube.

The four plates ( $L 1, L 2, L 3$ and $L 4)$ were of the same thickness of $1 \mathrm{~mm}$ and same orifice diameter of $2 \mathrm{~mm}$. The porosities of the individual plates, L1, L2, L3, L4 were respectively $1.40 \%, 3.14 \%, 5.59 \%$ and $7.07 \%$. This was achieved by changing the hole separation distance and the number of holes as shown in Figure 4. Each layer of perforated panel was backed by a $70 \mathrm{~mm}$ deep air cavity. The acoustic signal was a random noise signal and the incident sound pressure level was kept below $102 \mathrm{~dB}$ to avoid any non-linear absorption effect. Transient pressure data were collected and processed by the aforementioned two-microphone transfer function method in the experiments which will also be followed in the CFD simulations.

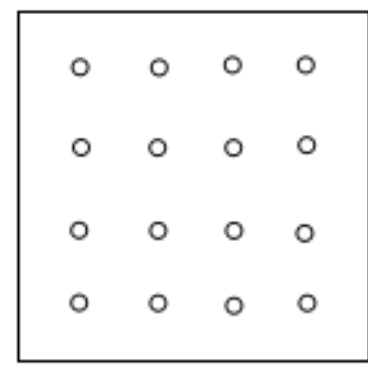

Plate L1

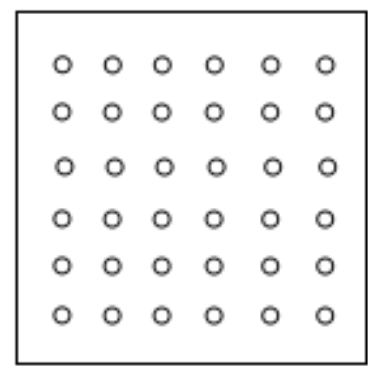

Plate L2

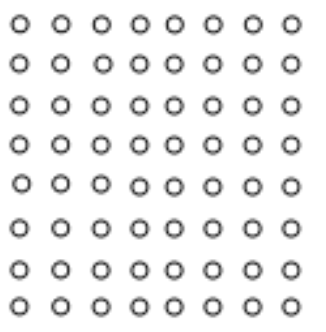

Plate L3

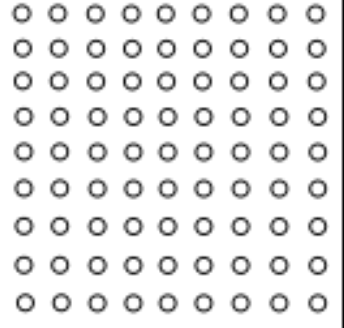

Plate L4

Figure 4 Picture of those perforated plates studied in Lee's experiment (7) 


\subsection{Non-Linear Absorption Regime}

The inertial resistance becomes significant in non-linear absorption regimes due to high particle velocities in the neck of the perforation hole. As a result, variations in incident sound pressure levels could alter the flow resistance and the overall noise attenuation effect of a plate absorber in the non-linear absorption regime. Past research has indicated that plates with smaller porosities tend to reach the non-linear absorption regime at lower sound pressure levels compared with those with a larger porosity $(19,30,41)$. Therefore, for a more convincing validation of the model, a perforated plate with a larger porosity was taken from the experiment by Melling (41) and a second orifice plate with a smaller porosity was taken from the experiment by Jing and Sun (17). Details of the two cases are shown below in Table 1.

\begin{tabular}{clccc}
\hline Plate & Porosity & Hole Diameter & Incident SPL & Orifice Thickness \\
\hline Melling RT335/00 & $7.6 \%$ & $2.8 \mathrm{~mm}$ & $116-160 \mathrm{~dB}$ & $0.56 \mathrm{~mm}$ \\
Jing and Sun & $0.5 \%$ & $8 \mathrm{~mm}$ & $96-144 \mathrm{~dB}$ & $0.1 \mathrm{~mm}$ \\
\hline
\end{tabular}

Table 1 Experimental parameters for the experiments of Melling (41) and Jing and Sun (17)

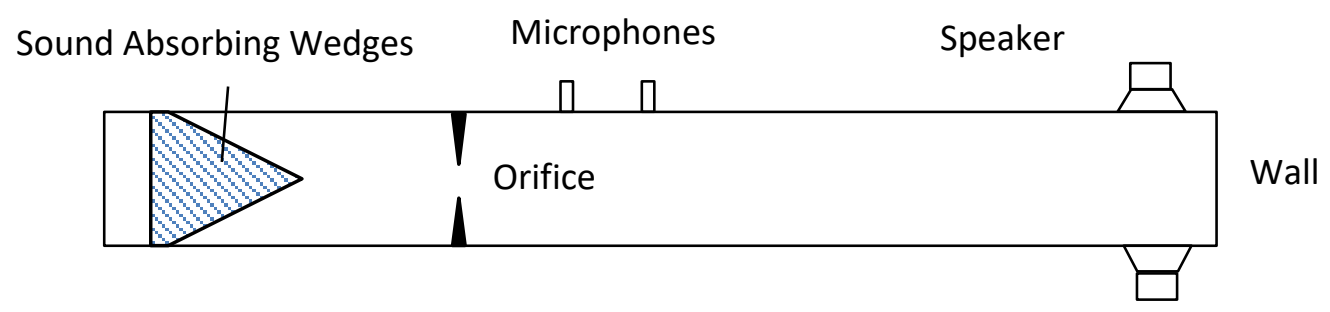

Figure 5 Experimental test rig setup in the experiment by Jing and Sun (17)

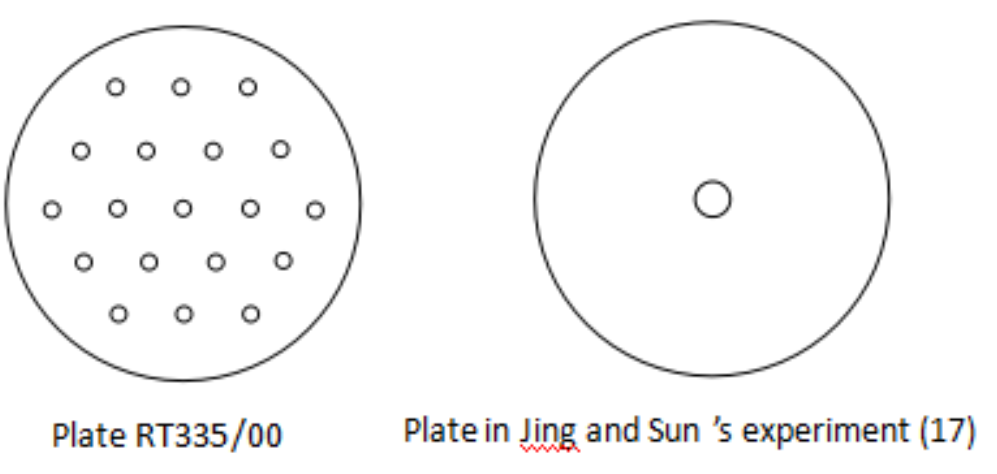

Figure 6 Picture of perforated plates studied in Melling's (41), Jing and Sun's experiment (17)

The experimental test rig in the experiment of Melling was again an impedance tube as shown in Figure 3. CFD calculations are carried out for the case where plate, indicated as RT335/00, was backed with a $25.4 \mathrm{~mm}$ deep cavity with an incident $1130 \mathrm{~Hz}$ sine-wave signal.

The experiment of Jing and Sun featured a single $\varnothing 8 \mathrm{~mm}$ circular orifice in the middle of a $100 \mathrm{~mm} \times 100 \mathrm{~mm}$ square plate as shown in Figure 5 . Downstream of the orifice, the cavity was filled with sound attenuation material so that acoustic wave reflection was negligible. The sound signal used in this experiment was a $200 \mathrm{~Hz}$ sine wave. Transient pressure data were collected and processed by the one-microphone transfer function method proposed by Chu (52). Melling's plate RT335/00 and the plate investigated in Jing and Sun's experiment are illustrated in Fig.6. 


\subsection{Bias Flow and Inertial Effect}

Jing et al. (10) investigated how the presence of bias flow affected acoustic properties of three perforated plate absorbers. Geometric specifications of those perforated plate absorbers investigated by Jing (10) are listed in Table 2 and shown in Figure 8. The impedance tube in Jing's work as shown in Figure 7 was a $100 \mathrm{~mm} \times 100 \mathrm{~mm}$ square tube in which sound signal of a wide frequency range $(100 \mathrm{~Hz}-1700 \mathrm{~Hz})$ could be tested. Further upstream of the impedance tube was equipped with an extension space and was filled with sound absorbing porous material so that incoming acoustic waves would not be bounced back to the impedance tube. Transient pressure data were collected and processed by the aforementioned two-microphone transfer function method in the experiments which will also be applied in their CFD simulations. Acoustic energy absorption coefficients were plotted against a resonance parameter $Q$ and which is a geometry dependent feature (6):

$$
Q=\frac{\pi}{2}\left(\mu k l_{c}\right)^{2}\left(\frac{r_{0}}{\mu k l_{c}}\right)
$$

where $\mu$ is dynamic viscosity of the air, $k$ is the signal wavenumber, $r_{0}$ is radius of orifices, $l_{c}$ is the cavity depth which is $20 \mathrm{~mm}$ or $60 \mathrm{~mm}$. The cross section of the cavity is a $120 \mathrm{~mm} \times 120 \mathrm{~mm}$ square.

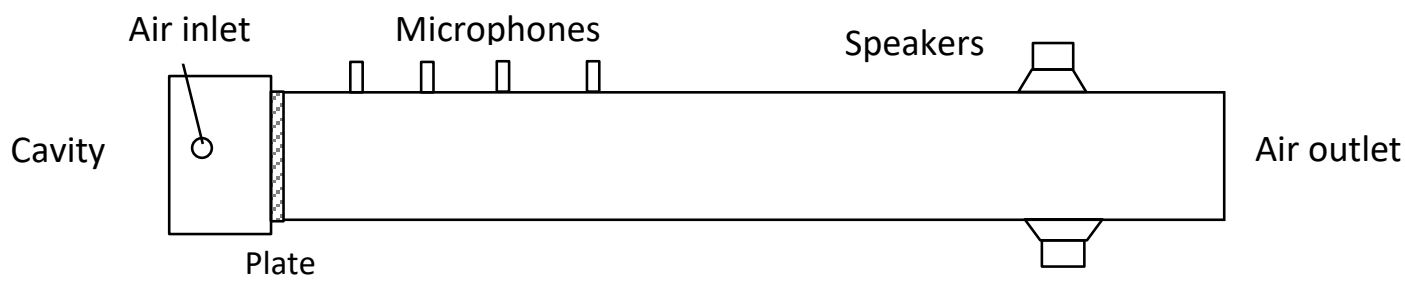

Figure 7 Impedance tube test rig setup in the experiments of Jing and Sun (10)

\begin{tabular}{rrrcc}
\hline & Orifice radius & Hole-Hole distance & Porosity & Thickness \\
\hline No.1 & $1.5 \mathrm{~mm}$ & $17 \mathrm{~mm}$ & 0.0254 & $0.2 \mathrm{~mm}$ \\
No.2 & $1.0 \mathrm{~mm}$ & $13 \mathrm{~mm}$ & 0.0201 & $0.2 \mathrm{~mm}$ \\
No.3 & $1.5 \mathrm{~mm}$ & $17 \mathrm{~mm}$ & 0.0254 & $2.0 \mathrm{~mm}$ \\
\hline
\end{tabular}

Table 2 Geometric specifications of plates in the experiment by Jing and Sun (10)
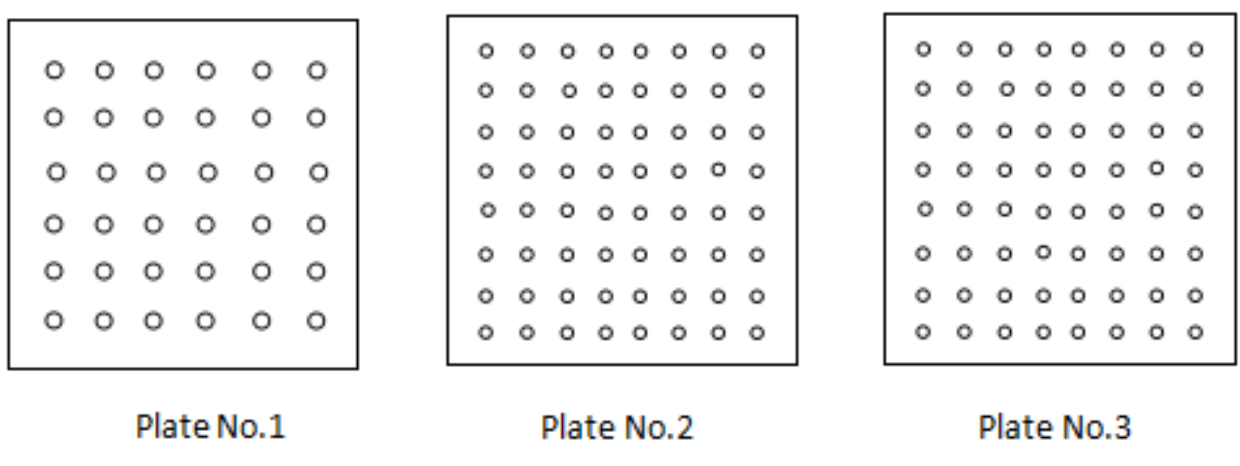

Figure 8 Picture of those perforated plates studied in the experiment by Jing and Sun (10) 


\section{Computational Simulation and Validation}

\subsection{Linear Absorption Regime}

Acoustic absorption coefficients of four perforated plate absorbers in the experiments by Lee and Kwon (7) were simulated by modelling the perforated plate using the PVPM model. The CFD results are compared with experimental results in Figure 9. Smoothing operation has been applied in both the experiment and CFD generated curves to remove minor random oscillations in the absorption data. As illustrated, the CFD generated absorption curves are in close agreement with the experimental results both in terms of resonance frequencies and the absorption bandwidth. CFD simulations were also carried out for two-layer L2 and three-layer L2 plate absorbers. The corresponding comparisons are shown in Figure 10 (a) and Figure 10(b). It is shown that the multilayer configuration reduces the first resonance frequency and the number of absorption effect peaks is the identical with the number of liner layers.

Figure 10(c) displays the absorption coefficient curve of an absorber made of three different plates in the sequence of L1-L2-L3 from upstream to downstream. By contrast, Figure 10(d) displays absorption coefficient of the absorber made of the same three-layer plates in the reverse order L3L2-L1. These two configurations yield very different attenuation effects. Large fluctuation exists in absorption coefficient curves of the former configuration while the latter configuration gives much smoother absorption curves. In addition, the L3-L2-L1 absorber setup yields much better absorption effect than the single layer L3 configuration as shown in Figure 4. CFD modelling indicates high levels of consistency with experimental data as all these features in experimental results are highlighted in the CFD results.
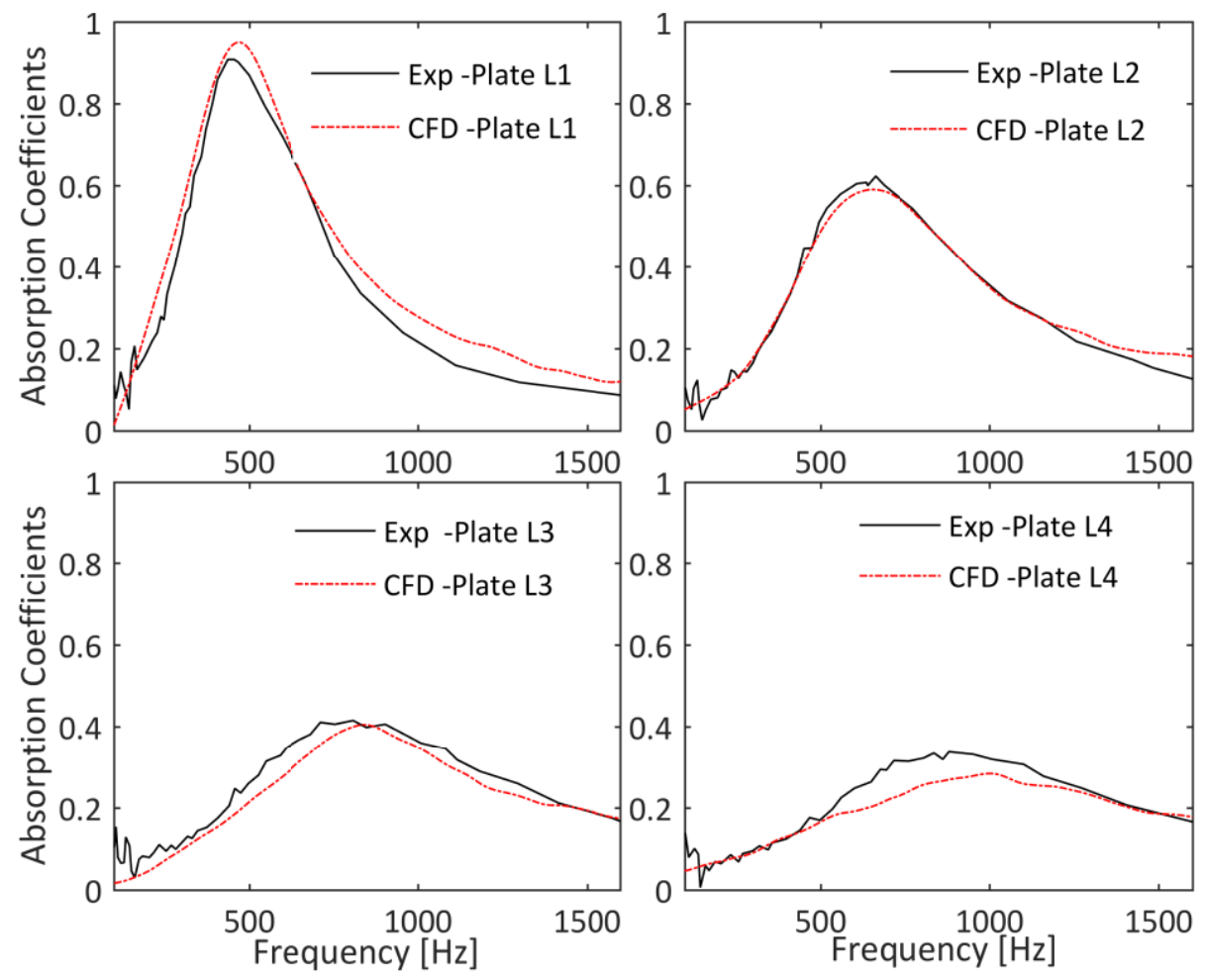

Figure 9 CFD and experimental comparison of absorption coefficients for single layer plate absorbers from the experiments of Lee and Kwon (7) 

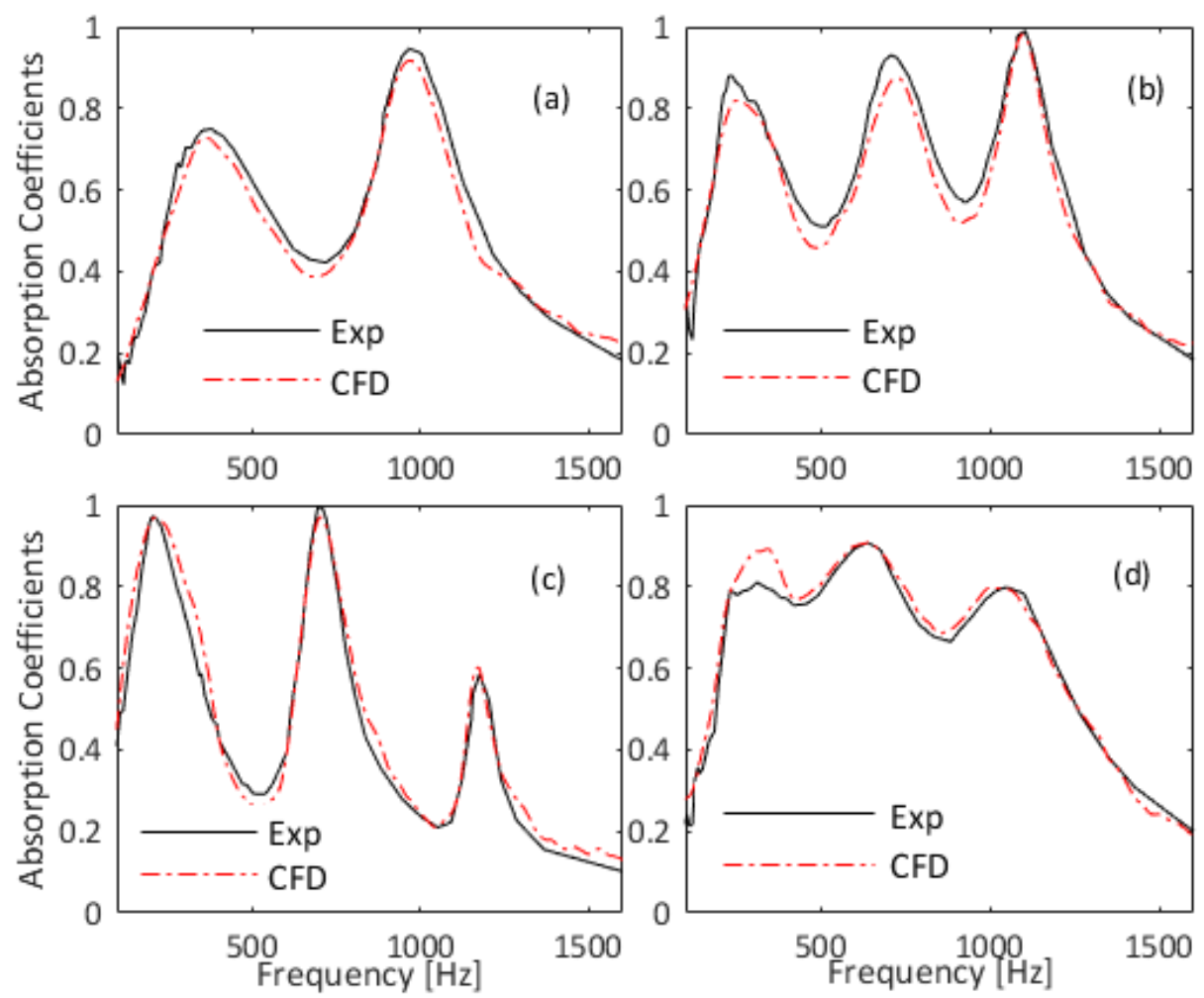

Figure 10 CFD and experimental comparison of absorption coefficients for multi-layer perforated panel absorbers: (a) 2-layer plate L2 (b) 3-layer plate L2 (c) 3-layer absorber of L1-L2-L3 (d) 3-layer absorber of L3-L2-L1 (7)

\subsection{Non-Linear Absorption Regime}

Figure 11 compares the normalized specific acoustic resistance of plate RT335/00 (41) obtained by CFD and experiment. CFD and experimental results agree closely and both methods show that the normalized specific acoustic resistance rises from a very low value (0.03) to approximately 1.5 as the incident sound pressure level rises from $118 \mathrm{~dB}$ to $160 \mathrm{~dB}$. Ingard and Ising (18) found in their experiment that acoustic specific resistance of an orifice due to high sound pressure levels is proportional to particle velocity amplitudes in the orifice. This finding was later confirmed by experiment by Jing et al. (17). Therefore, normalized resistance of an orifice in the presence of high particle velocities may be expressed as:

$$
r_{\text {orifice }}=\frac{\mathrm{C} \cdot U}{c}=\mathrm{C} \cdot \mathrm{M}
$$

Where M denotes Mach number of the neck particle velocity and the normalized specific acoustic resistance of the liner surface is:

$$
r_{\text {liner }}=\frac{\mathrm{C} \cdot U}{c \sigma}=\frac{\mathrm{C} \cdot \sqrt{2} \mathrm{RMS}\left(U_{\mathrm{ac}}\right)}{c \sigma}
$$

The normalized specific acoustic resistance for Melling's plate RT335/00 is calculated by imposing $\mathrm{C}=1$ in equation (22) using CFD acquired particle velocity and the result is plotted in Figure 11. Its agreement with experimental and numerical results is very close above SPL $130 \mathrm{~dB}$. Equation (22) assessed resistance is however somewhat lower than the experimental and numerically resistance at low sound pressure levels. This is because Ingard's linear equation is acquired at high sound pressure levels and tends to underestimate the contribution of viscous effect in low sound pressure levels. 


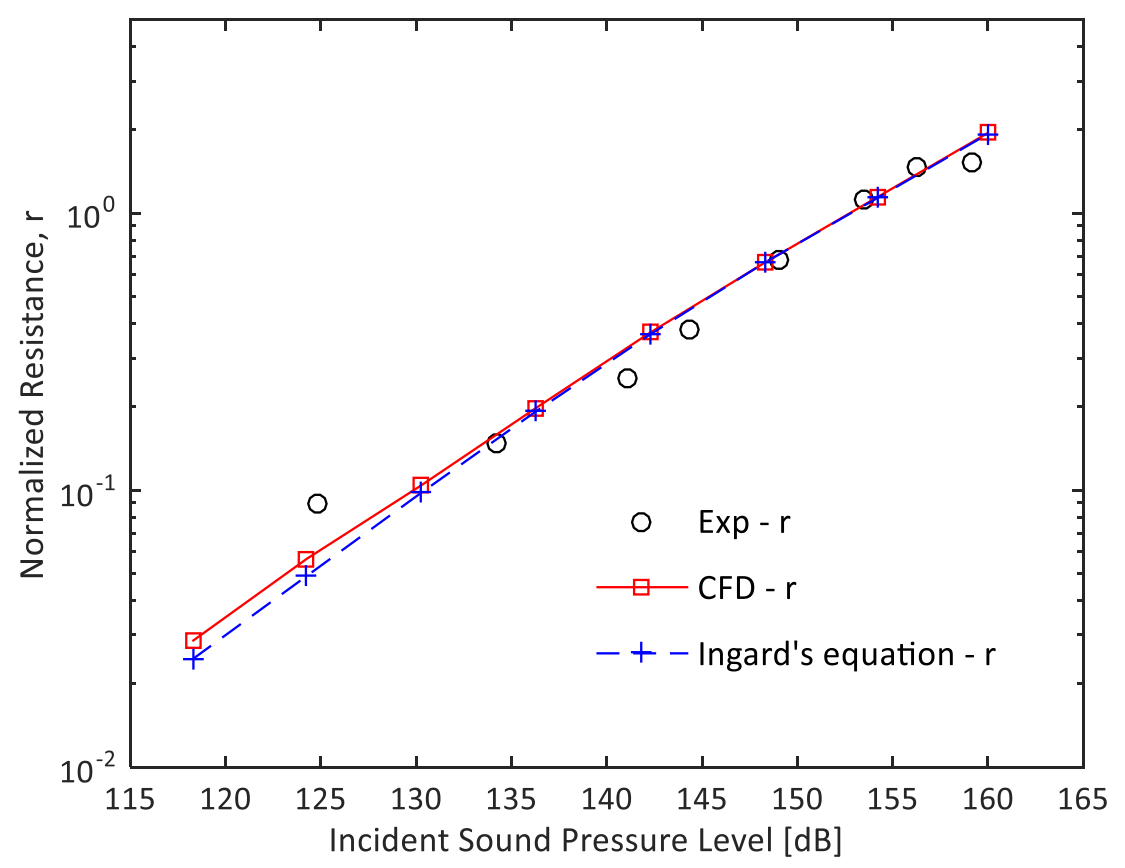

Figure 11 CFD and experimental comparison of normalized specific resistance of Plate RT335/00 in different incident sound pressure levels (41)

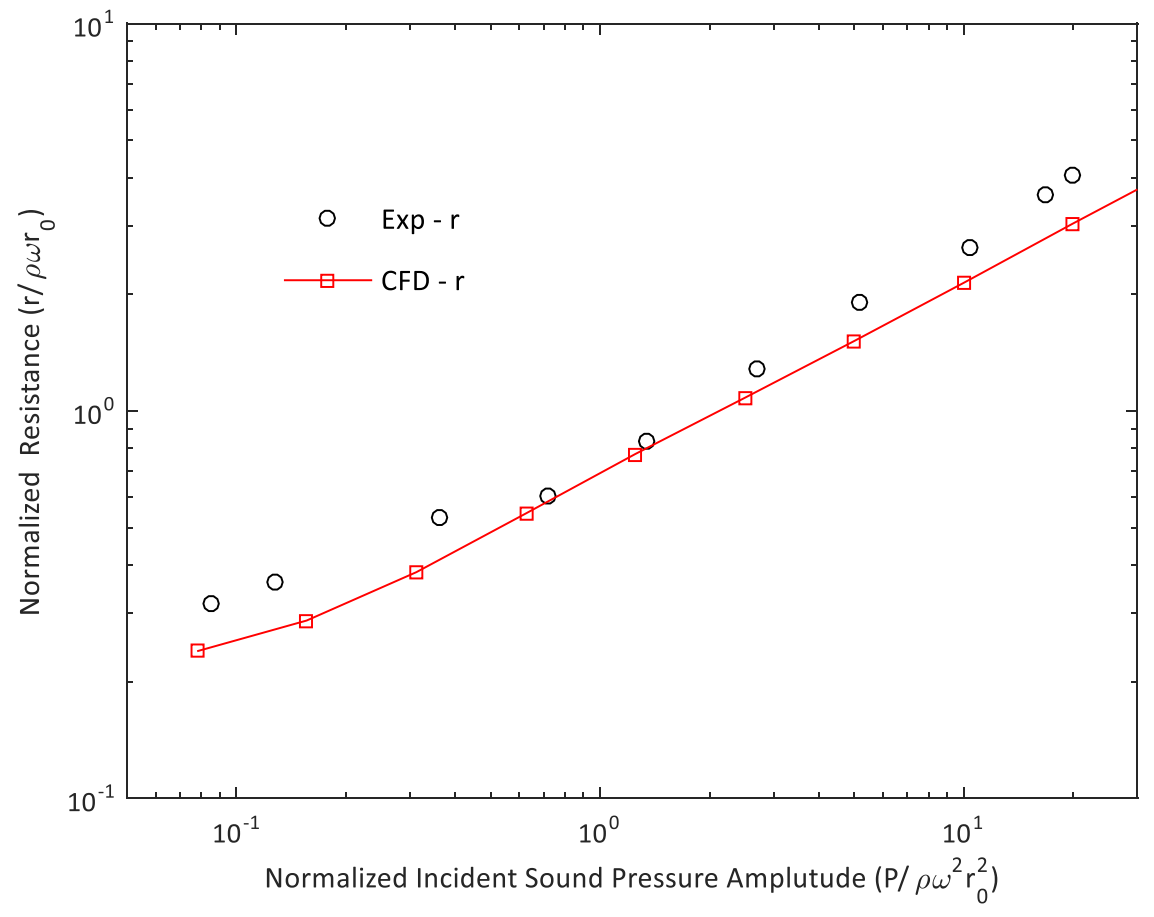

Figure 12 CFD and experiment results for Jing's orifice plate: change of normalized resistance with normalized incident sound pressure amplitude (17) 


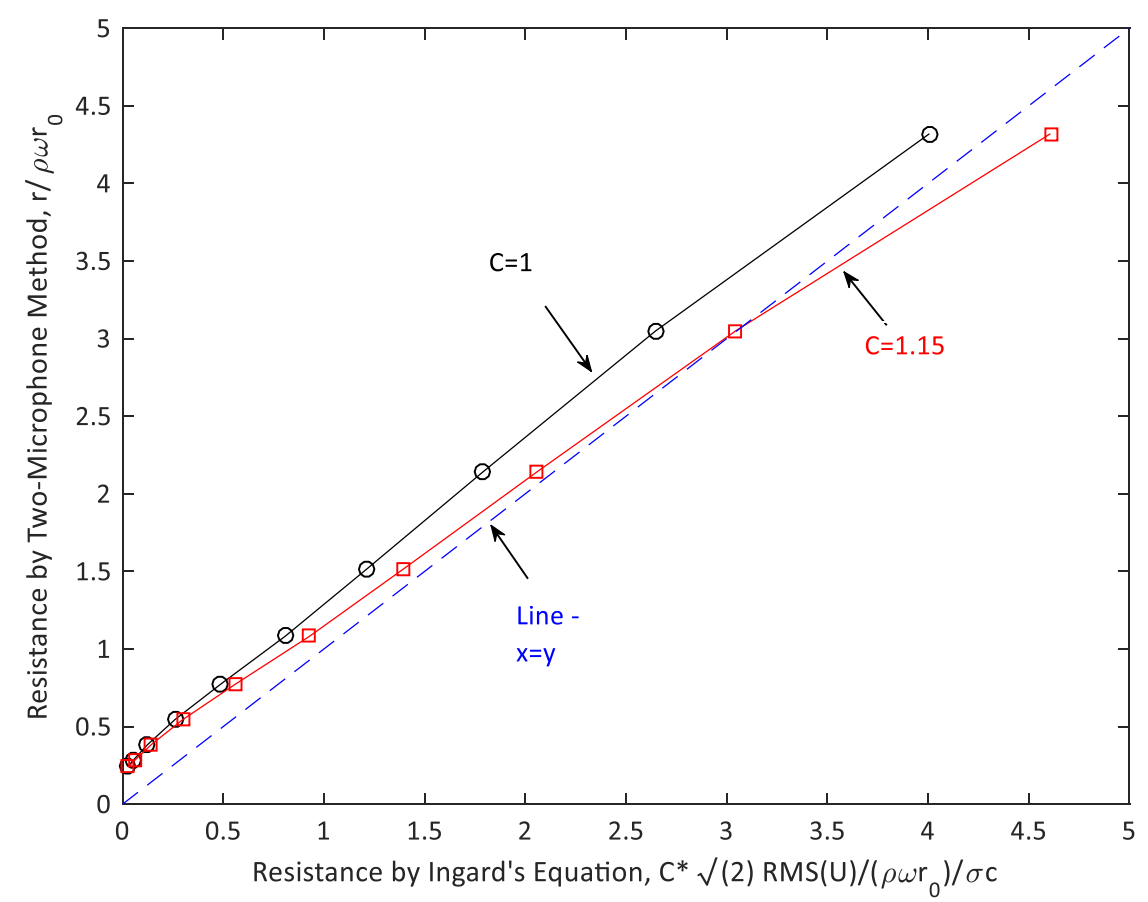

Figure 13 Determination of the proportional coefficient $C$ in Ingard's equation

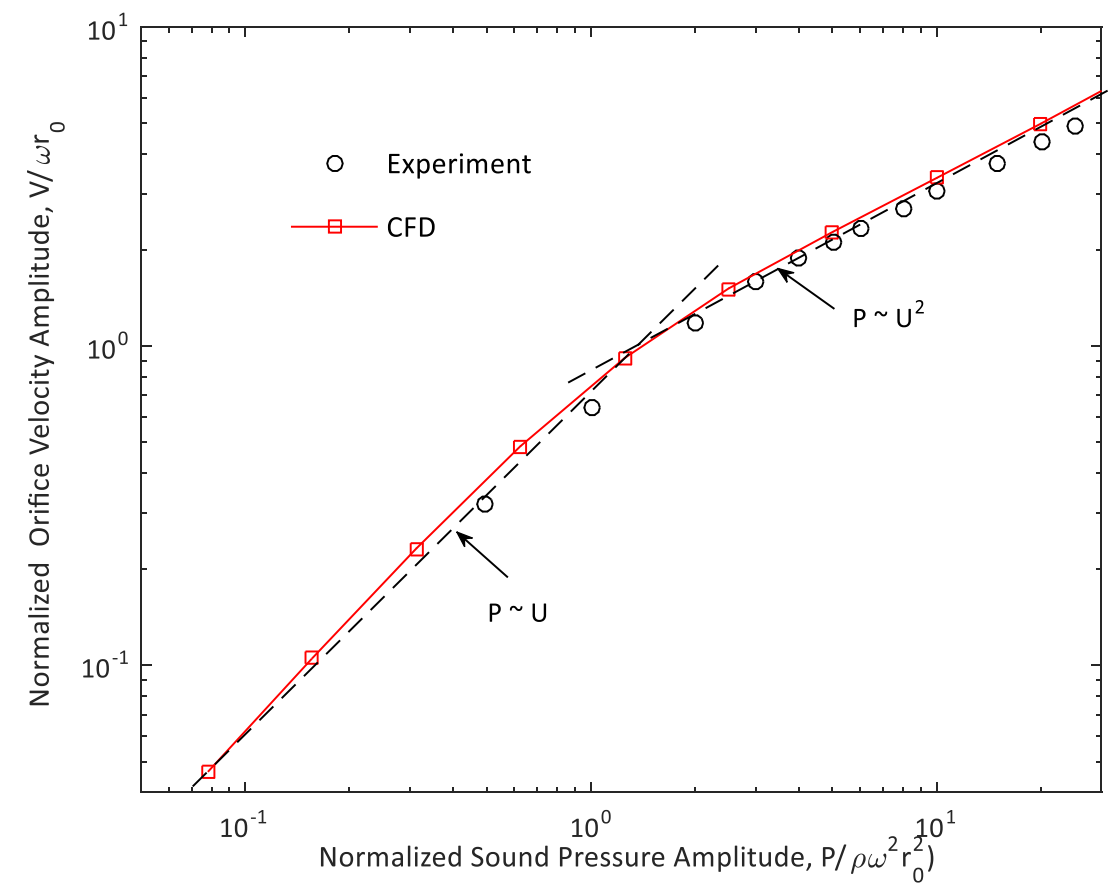

Figure 14 CFD and experimental comparison: change of orifice particle velocity with normalized incident sound pressure amplitude (17)

Figure 12 compares normalized acoustic resistance acquired for an orifice plate in the experiment of Jing et al (17) and by corresponding CFD simulations. Incidence sound pressure levels, specific acoustic impedance and orifice velocity are normalized by $\rho \omega^{2} \mathrm{r}_{0}^{2}, \rho \omega \mathrm{r}_{0}, \omega \mathrm{r}_{0}$ respectively, where $\omega$ is the incidence signal angular frequency, $\mathrm{r}_{0}$ is the radius of the orifice. CFD generated results again agree with the experimental results with quite satisfactory accuracy. Figure 13 plots normalized specific resistance acquired by equation (22) against that obtained by experimental and numerical 
method. As can be seen, imposing a higher coefficient $C=1.15$ in equation (22) mat ches CFD generated results more closely than $\mathrm{C}=1$ which is a more accurate value for Melling's plate.

Figure 14 plots how particle velocity in the perforated plate region changes with incident sound pressure amplitude. First, particle velocities obtained by CFD method agree very closely with those experimental results in a wide range of incident sound pressure amplitudes. Second, both methods indicate that particle velocity in the perforation is proportional to incident sound pressure amplitude within low incident sound pressure amplitudes. However, increase of particle velocity with incidence sound pressure amplitude slows down at high sound pressure levels and the pressure amplitude gradually changes to be in a quadratic relationship with particle velocity. This proves that the proposed CFD method is able to predict the resultant particle velocity due to a wide range of incident pressure amplitude and therefore is able to generate correct acoustic impedance within a wide range of incident sound pressure levels.

\subsection{Bias Flow and Inertial Effect}

Jing and Sun (10) experimentally tested acoustic properties of three perforated liners under various bias flow conditions. CFD and experimental absorption coefficient curves are compared in Figure 15 for an absorber made of plate No.1 under zero bias flow and $5 \mathrm{~m} / \mathrm{s}$ bias flow conditions. High degree of agreement between CFD and experiment is achieved in these two bias flow conditions. Both methods suggest that the presence of $5 \mathrm{~m} / \mathrm{s}$ bias flow is able to yield much higher absorption magnitude and broader absorption bandwidth. CFD and experimental results of normalized specific acoustic impedance for this absorber is compared in Figure 16. The reactance curve is flatter and zero reactance occurs at higher frequencies when $5 \mathrm{~m} / \mathrm{s}$ bias flow is present. According to equation (19), a flatter reactance curve and the higher frequency with zero reactance in $5 \mathrm{~m} / \mathrm{s}$ bias flow condition correspond well to Figure 15 where the absorption curve is broader and resonance frequency is higher in $5 \mathrm{~m} / \mathrm{s}$ bias flows. In addition, normalized specific resistance of the absorber increases from $0.2-0.3$ in zero flow condition to $1-1.2$ in $5 \mathrm{~m} / \mathrm{s}$ bias flow and this resistance rise is why absorption effect is noticeably enhanced by the presence of $5 \mathrm{~m} / \mathrm{s}$ bias flow.

Figure 17 displays the absorption effect of plate absorber made of plate No. 2 under several bias flow conditions. CFD results indicate that the noise attenuation effect rapidly rises with the introduction of bias flows. The absorption coefficient rises from approximately $50 \%$ in zero flow condition to $95 \%$ when bias flow Mach number is approximately 0.01 . Nearly $100 \%$ absorption is achieved at Mach number 0.012 . After this point, the absorption coefficient gradually declines but remains above $60 \%$ up to $\mathrm{M}_{\mathrm{b}}=0.04$. The experimental curve follows the same trend with CFD curves: absorption coefficient increases from $60 \%$ in zero flow condition to $100 \%$ at Mach number 0.012, it then declines at even higher bias flow Mach numbers. However, CFD method indicates somewhat better attenuation effects than the experiment does in low bias flow conditions. This error could potentially be exaggerated due to errors in the experimental absorption result which shows an abrupt change near Mach number 0.005 .

Figure 18 shows that both experiment and CFD indicate a linear acoustic resistance with bias flow velocities. The linear relationship between acoustic resistance and bias flow velocity is found by other researchers as well $(53,54)$.

$$
r=\frac{\mathrm{C}_{\mathrm{b}} \cdot U}{\sigma c}=\frac{\mathrm{C}_{\mathrm{b}} \cdot \mathrm{M}}{\sigma}
$$

The proportional constanct $C_{b}$ is related to contraction coefficients of the bias flow jet (54). The constant is approximately 1.5 for the current liner setup according to the CFD and experimental results shown in Figure 18. It is interesting to see the high similarity between equation (23) for mean bias flows and equation (22) for high sound incidence levels situations. The sole difference is that the proportional constant is higher in the presence of mean bias flow. 


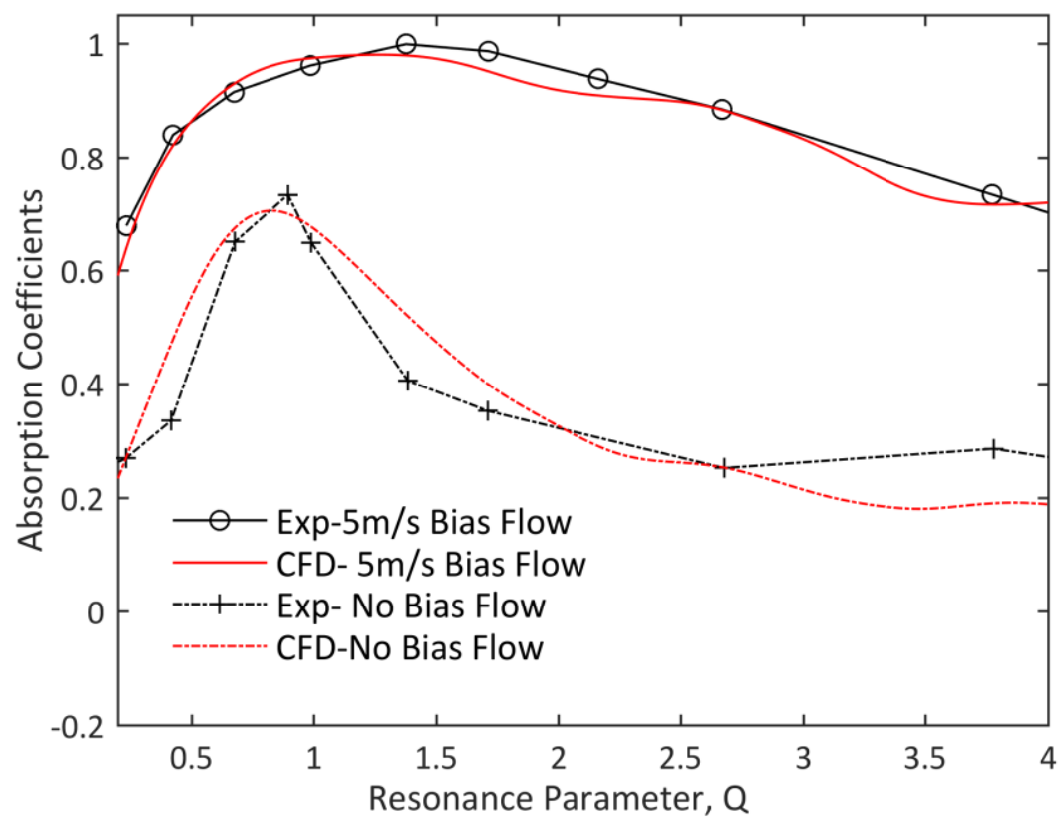

Figure 15 CFD and experimental comparison of absorption coefficients for perforated liner No.1 in $5 \mathrm{~m} / \mathrm{s}$ bias flow and zero bias flow conditions $\left(l_{\mathrm{c}}=60 \mathrm{~mm}\right)(10)$

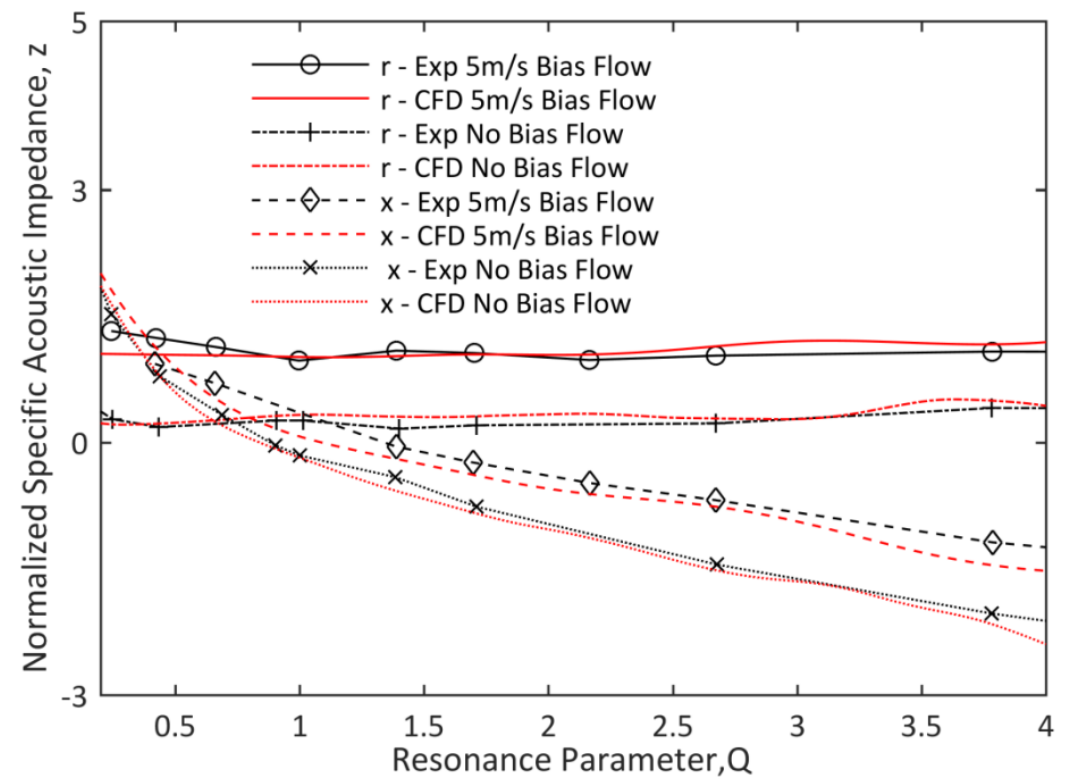

Figure 16 CFD and experimental comparison of normalized specific acoustic impedance for perforated liner No.1 in $5 \mathrm{~m} / \mathrm{s}$ bias flow and zero bias flow conditions $\left(l_{\mathrm{c}}=60 \mathrm{~mm}\right)(10)$ 


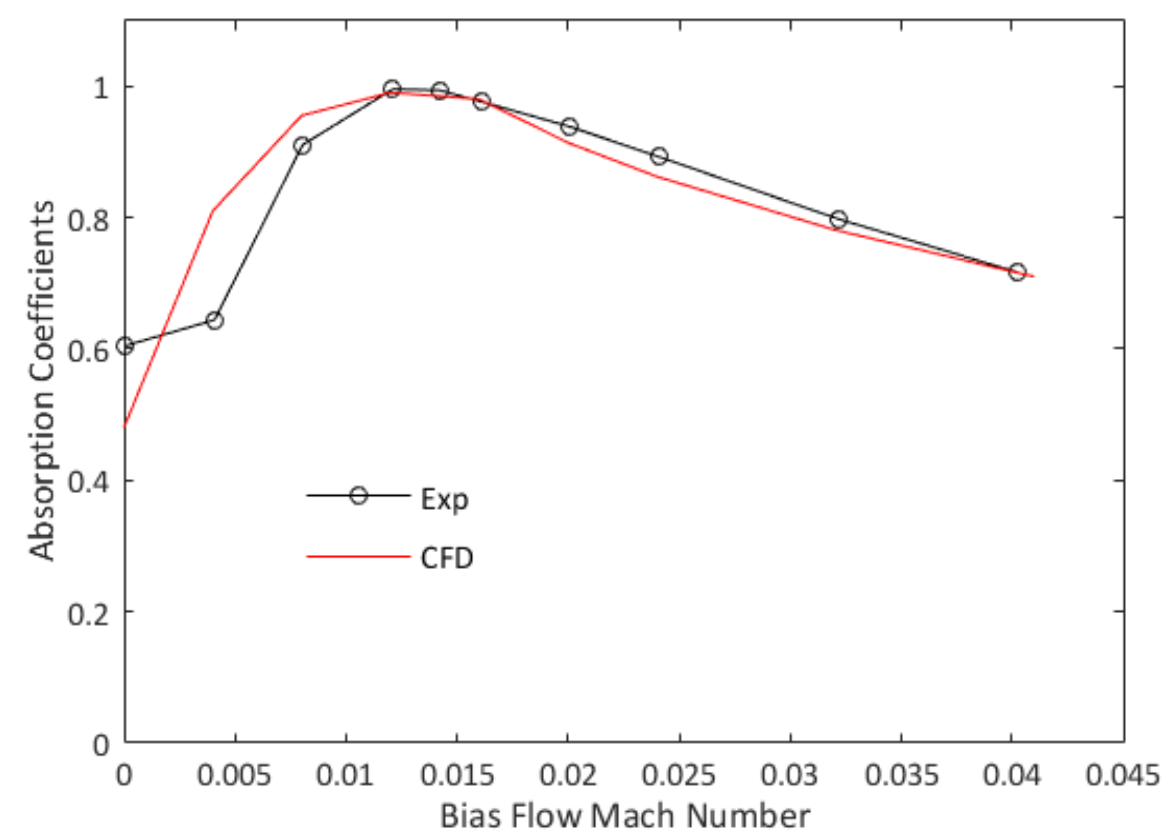

Figure 17 CFD and experimental comparison of absorption coefficients for perforated liner No.2 in different bias flow situations $\left(\mathrm{Q}=1, l_{\mathrm{c}}=60 \mathrm{~mm}\right)(10)$

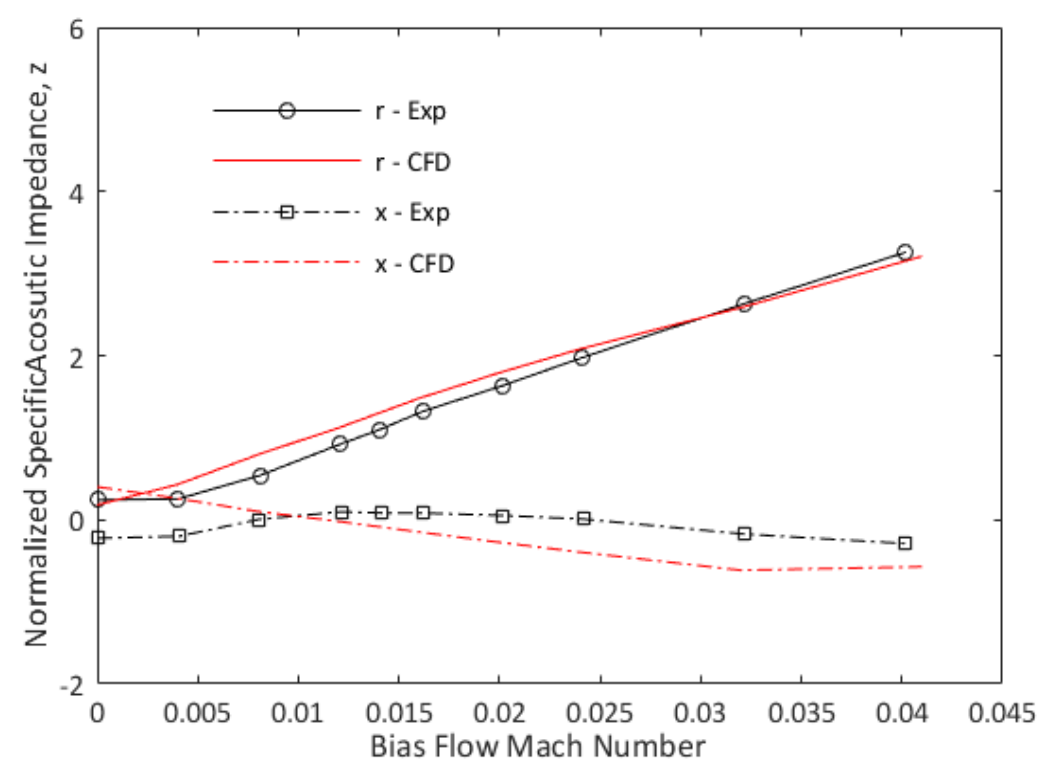

Figure 18 CFD and experimental comparison of normalized specific acoustic impedance for perforated liner No. 2 in different bias flow situations $\left(Q=1, l_{c}=60 \mathrm{~mm}\right)(10)$ 


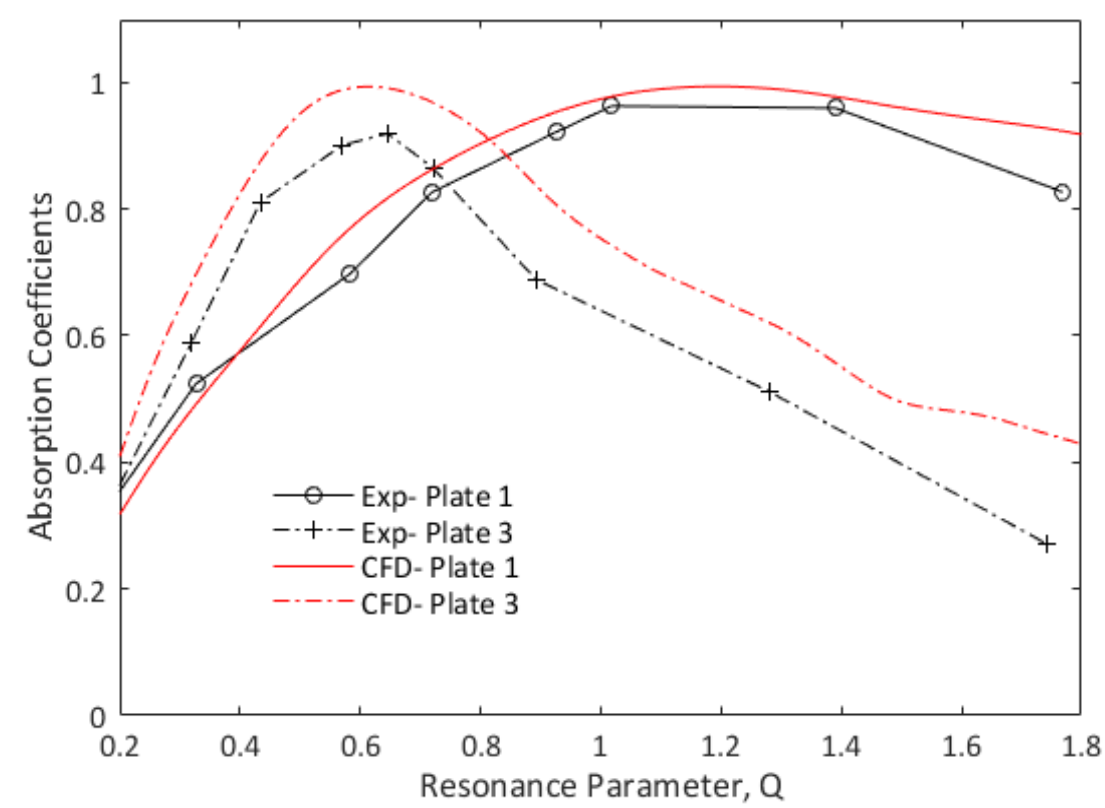

Figure 19 CFD and experimental comparison of absorption coefficients for perforated liners No.1 and No. 3 with different thickness under $5.4 \mathrm{~m} / \mathrm{s}$ bias flow $\left(l_{\mathrm{c}}=60 \mathrm{~mm}\right)(10)$

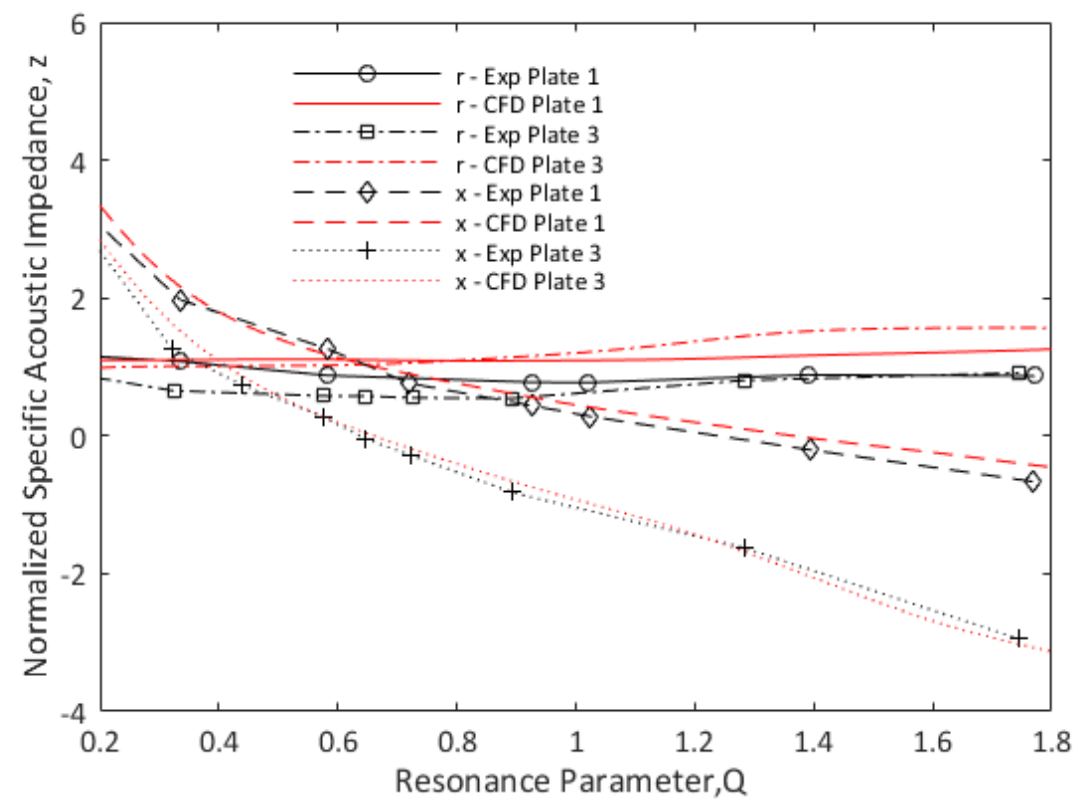

Figure 20 CFD and experimental comparison of normalized specific acoustic impedance for perforated liners No.1 and No.3 with different thickness under $5.4 \mathrm{~m} / \mathrm{s}$ bias flow condition

$$
\left(l_{c}=60 \mathrm{~mm}\right)(10)
$$

Figure 19 illustrates absorption coefficient curves acquired by CFD and experiment for perforated liners No.1 and No.3. The two liners No.1 and No.3 are with different thickness with all other plate features identical. Both CFD and experimental curves indicate that the thicker liner No.3 has a peak absorption effect at lower frequencies. In addition, the absorption coefficient curve for the thinner liner No.1 remains at high values within wider frequency range whereas the absorption curve for the thicker liner No.3 shows more rapid decline at high frequencies. This difference may be explained by considering the acoustic reactance curves displayed in Figure 20. The acoustic reactance curve of liner No.3 is steeper and zero reactance occurs at a lower frequency than that of liner No.1. According to equation (19), a steeper reactance curve and larger magnitudes of reactance will give 
rise to a narrower absorption coefficient curve according to equation (19). The absorption curves obtained by CFD and experiment for liner No.3 agree very well in terms of the general trend. However, errors exist as well, magnitude of CFD generated absorption coefficients are about 0.05 higher than those acquired by experiment within high frequencies.

In summary, CFD and experiments confirm that the introduction of bias flow enhances the noise attenuation effect of perforated liners. CFD is able to accurately predict the bias flow speeds at which optimum absorption effect is achieved. The effect of plate thickness on liner absorption property is represented by CFD with fairly good accuracy as well. In addition to the overall satisfactory agreement between CFD results and experimental results, there exists a limited degree of discrepancies as well in most CFD and experimental comparisons. These discrepancies could be caused by the fact that porous media model, by assuming homogeneous flow in a homogeneous region, overlooks the flow and geometric in-homogeneity near those orifices in a real perforated plate.

\section{Conclusion and Discussion}

In this paper the acoustic behaviour of a perforated plate was represented by a homogenous physical velocity porous media (PVPM) model. The model was demonstrated to be able to significantly reduce the grid resolution in comparison to detailed geometric resolution of a perforated plate, whilst being able to accurately simulate the acoustic behaviour under normal signal incidence conditions in an impedance tube. Experimental results from a number of wellacknowledged acoustic experiments were reproduced by the proposed CFD method. Comparison between three dimensional, compressible, laminar flow CFD simulations and experimental data, demonstrated that the porous model is able to correctly represent the acoustic properties of perforated plate absorbers. This was demonstrated for both linear and non-linear absorption regimes and also for the inertial effect in the presence of a mean bias flow.

Application of this model will provide a computationally efficient method for the CFD simulation of multi-perforated plates. The model significantly reduces the computational resource required in comparison to full geometric resolution and is thus a promising tool for the engineering design of perforated plate absorbers.

\section{Acknowledgement}

The University of Hull and China Scholarship Council are greatly acknowledged for the financial support in making this research possible.

\section{References}

1. Lieuwen T, Neumeier $\mathrm{Y}$, Zinn BT. The role of unmixedness and chemical kinetics in driving combustion instabilities in lean premixed combustors. Combustion Science and Technology. 1998;135(1-6):193-211.

2. Venkataraman KK, Preston LH, Simons DW, Lee BJ, Lee JG, Santavicca DA. Mechanism of combustion instability in a lean premixed dump combustor. Journal of Propulsion and Power. 1999;15(6):909-18.

3. Lefebvre AH. Gas turbine combustion: Heat transfer: CRC press; 1998.

4. Farmer G, Brown DD, Rutherford ME, inventors; Google Patents-US6655149, assignee. Preferential multihole combustor liner. USA,2003. 
5. Jing $X$, Sun $X$. Effect of plate thickness on impedance of perforated plates with bias flow. AIAA Journal. 2000;38(9):1573-8.

6. Hughes IJ, Dowling AP. The absorption of sound by perforated linings. Journal of Fluid Mechanics. 1990;218:299-335.

7. Lee DH, Kwon YP. Estimation of the absorption performance of multiple layer perforated panel systems by transfer matrix method. Journal of sound and vibration. 2004;278(4):847-60.

8. Jung SS, Kim YT, Lee DH, Kim HC, Cho SI, Lee JK. Sound absorption of micro-perforated panel. Journal of the Korean Physical Society. 2007;50(4):1044.

9. Houston B, Wang J, Qin Q, Rubini P. Experimental and numerical investigation of Helmholtz resonators and perforated liners as attenuation devices in industrial gas turbine combustors. Fuel. 2015;151:31-9.

10. Jing X, Sun X. Experimental investigations of perforated liners with bias flow. The Journal of the Acoustical Society of America. 1999;106(5):2436-41.

11. Chanaud RC. Effects of geometry on the resonance frequency of Helmholtz resonators. Journal of Sound and Vibration. 1994;178(3):337-48.

12. Grace SM, Horan KP, Howe MS. The influence of shape on the Rayleigh conductivity of a wall aperture in the presence of grazing flow. Journal of Fluids and Structures. 1998;12(3):335-51.

13. Laurens S, Tordeux S, Bendali A, Fares M, Kotiuga PR. Lower and upper bounds for the Rayleigh conductivity of a perforated plate. ESAIM: Mathematical Modelling and Numerical Analysis. 2013;47(6):1691-712.

14. Rupp J, Carrotte J, Macquisten M. The use of perforated damping liners in aero gas turbine combustion systems. Journal of Engineering for Gas Turbines and Power. 2012;134(7):071502.

15. Zhao D, Ang L, Ji CZ. Numerical and experimental investigation of the acoustic damping effect of single-layer perforated liners with joint bias-grazing flow. Journal of Sound and Vibration. 2015;342:152-67.

16. Liu J, Herrin DW. Enhancing micro-perforated panel attenuation by partitioning the adjoining cavity. Applied Acoustics. 2010;71(2):120-7.

17. Jing $X$, Sun $X$. Sound-excited flow and acoustic nonlinearity at an orifice. Physics of Fluids (1994-present). 2002;14(1):268-76.

18. Ingard $\mathrm{U}$, Ising $\mathrm{H}$. Acoustic nonlinearity of an orifice. The journal of the Acoustical Society of America. 1967;42(1):6-17.

19. Tayong R, Dupont T, Leclaire P. On the variations of acoustic absorption peak with particle velocity in micro-perforated panels at high level of excitation. The Journal of the Acoustical Society of America. 2010;127(5):2875-82.

20. Eldredge JD, Dowling AP. The absorption of axial acoustic waves by a perforated liner with bias flow. Journal of Fluid Mechanics. 2003;485:307-35.

21. Sun $X$, Jing $X$, Zhang $H$, Shi Y. Effect of grazing-bias flow interaction on acoustic impedance of perforated plates. Journal of Sound and Vibration. 2002;254(3):557-73.

22. Jing X, Sun X, Wu J, Meng K. Effect of grazing flow on the acoustic impedance of an orifice. AIAA Journal. 2001;39(8):1478-84.

23. Kirby $R$, Cummings $A$. The impedance of perforated plates subjected to grazing gas flow and backed by porous media. Journal of Sound and Vibration. 1998;217(4):619-36.

24. Dassé J, Mendez S, Nicoud F. Large-eddy simulation of the acoustic response of a perforated plate. 14th AIAA/CEAS Aeroacoustics Conference (29th AIAA Aeroacoustics Conference); 05 May 07 May 2008; Vancouver,Canada2008.

25. Boudier G, Gicquel LYM, Poinsot T, Bissieres D, Bérat C. Comparison of LES, RANS and experiments in an aeronautical gas turbine combustion chamber. Proceedings of the Combustion Institute. 2007;31(2):3075-82.

26. Fan W, Guo L-X. An Investigation of Acoustic Attenuation Performance of Silencers with Mean Flow Based on Three-Dimensional Numerical Simulation. Shock and Vibration. 2016;2016. 
27. Liu C, Ji Z. Computational fluid dynamics-based numerical analysis of acoustic attenuation and flow resistance characteristics of perforated tube silencers. Journal of Vibration and Acoustics. 2014;136(2):021006.

28. Zhang $Q$, Bodony DJ. Numerical investigation and modelling of acoustically excited flow through a circular orifice backed by a hexagonal cavity. Journal of Fluid Mechanics. 2012;693:367401.

29. Mendez S, Eldredge JD. Acoustic modeling of perforated plates with bias flow for large-eddy simulations. Journal of Computational Physics. 2009;228(13):4757-72.

30. Lahiri C. Acoustic performance of bias flow liners in gas turbine combustors. Berlin, Germany: Technische Universität Berlin; 2014.

31. Gaeta RJ, Ahuja KK. Effect of orifice shape on acoustic impedance. International Journal of Aeroacoustics. 2016;15(4-5):474 - 95.

32. Andreini A, Facchini B, Ferrari L, Lenzi G, Simonetti F, Peschiulli A, editors. Experimental investigation on effusion liner geometries for aero-engine combustors: evaluation of global acoustic parameters. ASME Turbo Expo 2012: Turbine Technical Conference and Exposition; 2012 2012; Copenhagen, Denmark: American Society of Mechanical Engineers.

33. Sivertsen BR, Djilali N. CFD-based modelling of proton exchange membrane fuel cells. Journal of Power Sources. 2005;141(1):65-78.

34. Gage KL, Gartner MJ, Burgreen GW, Wagner WR. Predicting membrane oxygenator pressure drop using computational fluid dynamics. Artificial organs. 2002;26(7):600-7.

35. Endalew AM, Hertog M, Delele MA, Baetens K, Persoons T, Baelmans M, et al. CFD modelling and wind tunnel validation of airflow through plant canopies using 3D canopy architecture. International Journal of Heat and Fluid Flow. 2009;30(2):356-68.

36. Deutschmann O. Computational fluid dynamics simulation of catalytic reactors. Handbook of heterogeneous catalysis. 2008.

37. Sutera SP, Skalak R. The history of Poiseuille's law. Annual Review of Fluid Mechanics. 1993;25(1):1-20.

38. Ingard U. On the theory and design of acoustic resonators. The Journal of the Acoustical Society of America. 1953;25(6):1037-61.

39. Rayleigh L. Theory of Sound, Vol. II, 322 i. Macmillan; 1940.

40. Fok, Brocklesby MW. Theoretical study of the conductance of a circular hole in a partition across a tube. Doklady Akademii Nauk SSSR. 1941;31(9):875-82.

41. Melling TH. The acoustic impedance of perforates at medium and high sound pressure levels. Journel of Sound and Vibration. 1973;29:1-65.

42. Ingard U. Attenuation and regeneration of sound in ducts and jet diffusers. The Journal of the Acoustical Society of America. 1959;31(9):1202-12.

43. Maa D-Y. Microperforated panel at high sound intensity [J]. Acta Acustica. 1996;1.

44. Dean PD, Tester BJ. Duct wall impedance control as an advanced concept for acoustic impression. Marietta, United States; 1975.

45. Elnady T. Modelling and characterization of Perforates in Lined Ducts and Mufflers. Stockholm: KTH Royal Institute of Technology in Stockholm; 2004.

46. Poinsot TJ, amp, Lelef SK. Boundary conditions for direct simulations of compressible viscous flows. Journal of Computational Physics. 1992;101(1):104-29.

47. Selle L, Nicoud F, Poinsot T. Actual impedance of nonreflecting boundary conditions: Implications for computation of resonators. AIAA Journal. 2004;42(5):958-64.

48. Gerke HH, Genuchten MTV. A dual-porosity model for simulating the preferential movement of water and solutes in structured porous media. Water Resources Research. 1993;29(2):305-19.

49. Mualem Y. A new model for predicting the hydraulic conductivity of unsaturated porous media. Water Resources Research. 1976;12(3):513-22.

50. Champoux Y, Allard JF. Dynamic tortuosity and bulk modulus in air - saturated porous media. Journal of Applied Physics. 1991;70(4):1975-9. 
51. Seybert AF, Ross DF. Experimental determination of acoustic properties using a two microphone random - excitation technique. the Journal of the Acoustical Society of America. 1977;61(5):1362-70.

52. Chu WT. Transfer function technique for impedance and absorption measurements in an impedance tube using a single microphone. The Journal of the Acoustical Society of America. 1986;80(2):555-60.

53. Sivian LJ. Acoustic impedance of small orifices. The Journal of the Acoustical Society of America. 1935;7(2):94-101.

54. Cummings A, Eversman W. High amplitude acoustic transmission through duct terminations: Theory. Journal of Sound and Vibration. 1983;91(4):503-18. 